\title{
Grain-boundary scattering in semiconductor films
}

\begin{abstract}
A K PAL
Department of Materials Science, Indian Association for the Cultivation of Science, Calcutta 700032 , India

Abstract. Alternative technique for studying grain-boundary scattering phenomena in high-resistivity semiconducting films has been indicated. The combined effect of the presence of electric field and mechanical stress at the grain boundaries was considered. It is shown that the grain boundary potential, density of trap states, and carrier concentration of the films can be obtained by measuring reflectances of the films deposited on non-absorbing substrates.
\end{abstract}

Keywords. Grain-boundary scattering; semiconductor; polycrystalline film; Franz-Keldysh effect.

\section{Introduction}

Most of the devices based on compound semiconductors are generally polycrystalline in nature where the films are composed of a large number of crystallites joined together by grain boundaries. The intercrystalline boundaries or grain boundaries are one of the most dominant factors controlling conduction mechanism in polycrystalline semiconductor films. In electronic devices and solar cells based either on elemental or compound semiconductors, the deleterious effects of grain boundaries are well known. Several models have been proposed by workers in this field to describe grain-boundary phenomena in polycrystalline films (Volger 1950; Kamins 1971; Seto 1975, 1976; Baccarani et al 1978). All the methods involved measuring either electrical conductivity $(\sigma)$ or Hall mobility $(\mu)$ at lower temperatures, when the contributions due to phonons to the total scattering is less. However, it is seen that the films used for device fabrication are generally of high resistivity and as such measurement of $\sigma$ and specially $\mu$ becomes difficult, if not impossible, at lower temperatures. So there is a need for an alternative route for studying the grainboundary effect in highly resistive films. Measurements of optical reflectances have offered such a possibility.

The effect of grain boundaries with an inherent space charge region due to the interface results in band bending. This is reflected in the departure from the sharpness of the fall of absorption coefficient $(\alpha)$ near the absorption edge. This culminates in the appearance of an additional absorption at longer wavelength. The amount of additional absorption depends on the grain size of the film.

The effects of grain boundaries on the electronic structure and optical properties of a polycrystalline semiconductor are mainly determined by the following three factors:

(i) Physicochemical imperfections: They arise from atomic bond angle and bond length deviations. 
(ii) Geometrical imperfections: (a) External electric fields arising as a result of the screening of the charge near the grain-boundary region, and (b) the presence of mechanical stresses due to structural defects and dislocations at the grain-boundary regions.

However, for polycrystalline films with moderate grain size, the contribution of the physicochemical imperfections to the grain-boundary effect is quite negligible (Gavrilenko 1987). In this case, geometrical imperfections mainly govern the electronic and optical properties. Several attempts (Redfield 1963; Dow and Redfield 1970; Bujatti and Marcelja 1972; Bugnet 1974; Szczyrbowsky and Czapla 1977) were made to describe the optical absorption in polycrystalline films. Among them, the DowRedfield model (Redfield 1963, 1965; Dow and Redfield 1970, 1972), which assumes that the excess absorption is due to Franz-Keldysh effect arising out of the electric field present in the material, seems to be a realistic approach towards understanding the absorption behaviour at the band edge due to grain boundaries. We present here a modified treatment for evaluating the grain-boundary scattering effects in polycrystalline films as presented by Dow-Redfield by considering the effects of surface roughness that may prejudice the determination of the absorption coefficient in polycrystalline films (Bhattacharyya et al 1992a,b). We also considered the effect of mechanical stress present at the grain boundaries on the absorption coefficient for the above films. The experimental data were fitted by considering all the possibilities to find out conditions of the best fit. This method allows one to evaluate the grainboundary barrier height $\left(E_{\mathrm{b}}\right)$, density of trap states $\left(Q_{\mathrm{t}}\right)$ and carrier concentration $\left(N_{\mathrm{d}}\right)$ in high-resistivity films where determination of the above quantities by conventional techniques is extremely difficult.

\section{Theory}

\subsection{Franz-Keldysh effect}

The band bending near the absorption edge and the broadening of the tail end of the absorption spectrum ( $\alpha-h v$ plot) of polycrystalline semiconductor films mainly arise out of the contributions from three different phenomena: band-to-band absorption beyond the fundamental band edge, defect and impurity absorption, and an excess absorption caused by the Franz-Keldysh effect arising out of the built-in electric field in the material. The Franz-Keldysh effect is basically phonon-assisted tunnelling which considers that in the presence of an electric field the electron has a non-zero probability of being in the forbidden gap. This allows a photon to cause a transition to the conduction band at energies less than the bandgap $\left(E_{\mathrm{g}}\right)$. Based on this basic assumption, Dow and Redfield $(1970,1972)$ obtained an expression for $\alpha$ containing the field $(F)$ and the expression was subsequently modified by Bujatti and Marcelja (1972) and Bugnet (1974).

Bujatti and Marcelja modified the Dow-Redfield model by assuming spherical crystallites with some distribution $P(R)$ of the radius $(R)$ of the spheres. They could obtain a useful expression for the normalized total absorption $(A)$ from which the potential barriers at the grain boundaries could be obtained under some simplified assumptions concerning the shape of the barrier. Using the subscript zero to indicate quantities at the energy gap, the normalized total absorption was expressed as (Bujatti 
and Marcelja 1972)

$$
A / A_{0}=\frac{\int_{0}^{\infty} P(R) \mathrm{d} R \int_{0}^{R} \alpha^{E}(F, \hbar \omega) r^{2} \mathrm{~d} r}{\int_{0}^{\infty} P(R) \mathrm{d} R \int_{0}^{R} \alpha_{0}^{E} r^{2} \mathrm{~d} r},
$$

where $P(R)$ is the probability for a crystallite to have a radius $R$. It can be noted here that $\alpha$ appearing in (1) is a function of both photon energy and electric field in the crystallites. $\alpha^{E}(F, \hbar \omega)$ may be expressed in its generalized form as

with

$$
\alpha^{E}(F, \hbar \omega)=I(F, \hbar \omega) F^{1 / 3},
$$

and

$$
\begin{aligned}
& I(F, \hbar \omega)=K \omega^{-1} \int_{y}^{\infty} A_{i}^{2}(z) \mathrm{d} z, \\
& K=\frac{4 \mu e^{4}\left|<P^{2}>\right|}{m_{0}^{2} \hbar^{2} n \varepsilon_{0} c} \gamma^{1 / 3}, \\
& \left|<P^{2}>\right|=\frac{m_{0} E_{\mathrm{g}}\left(E_{\mathrm{g}}+\Delta_{0}\right)}{12\left(E_{\mathrm{g}}+2 \Delta_{0} / 3\right)}\left(\frac{m_{0}}{m_{\mathrm{e}}}-1\right), \\
& \mu=\left[m_{\mathrm{e}} m_{\mathrm{h}} /\left(m_{\mathrm{e}}+m_{\mathrm{h}}\right)\right] ; \gamma=\left(2 \mu / \hbar^{2}\right),
\end{aligned}
$$

$$
y=\gamma^{1 / 3} F^{-2 / 3}\left(E_{\mathbf{g}}-h v\right) \text {. }
$$

Here $m_{0}, m_{\mathrm{c}}$ and $m_{\mathrm{h}}$ are the free and effective masses of electron and hole respectively, $\Delta_{0}$ the spin-orbit splitting, $n$ the refractive index, and $c$ the velocity of light. The subscript zero indicates quantities at the energy gap $\left(E_{\mathrm{g}}\right) . \varepsilon_{0}$ is the permittivity of free space.

For polycrystalline films with large crystallites, the surface states are, in general, all occupied by electrons and the electric field close to the surface of the grains decays exponentially as shown in figure 1 . We assumed here a field distribution in the

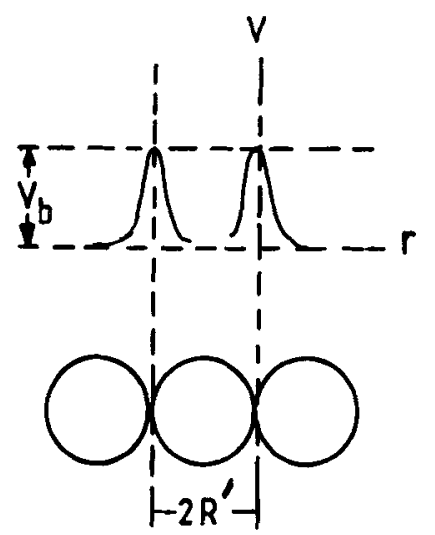

Figure 1. Potential form at the grain boundaries with different grain size. 
following form:

$$
F=F_{\mathrm{s}} \exp [-k(R-r)]
$$

where $F_{\mathrm{s}}\left(=\frac{e^{2} Q_{\mathrm{t}}}{\varepsilon}\right)$ is the ratio of surface charge density and static dielectric constant, $k\left[=\left\{\left(4 \pi \varepsilon k_{\mathrm{b}} T\right) / N_{\mathrm{d}} e^{2}\right\}^{-1 / 2}\right]$ the inverse of the Debye screening length $\left(\lambda_{\mathrm{d}}\right), Q_{\mathrm{t}}$ the density of trap states at the grain boundaries, and $N_{\mathrm{d}}$ the carrier concentration. This form of field distribution is a generalized one which can describe both partially and fully depleted conditions of the grains (Bhattacharyya et al 1993). The depletion at the grain-boundary regions results in band bending and consequently in optical absorption below the band-edge region. In order to include the contribution of different grain sizes in $\alpha$, we have assumed a distribution of grain size having spherical nature of the form given by (Bhattacharyya et al 1992b)

$$
P(R)=\left(R-R_{\min }\right)\left(R_{\max }-R\right) \exp \left(-R^{2} / \delta^{2}\right),
$$

where $R_{\max }=\bar{R}+\delta$ and $R_{\min }=\bar{R}-\delta, \bar{R}=\left(R_{\max }+R_{\min }\right) / 2$, and $\delta(=0 \cdot 76 \bar{R})$ is the half-width of the distribution of $R$. This probability function $P(R)$ increases rapidly for small values of $R$ and then decreases with a Gaussian tail for large values of $R$.

Using (2) to (6) the final form of 1 becomes:

$$
A / A_{0}=\alpha^{E}(\hbar \omega) / \alpha_{0}^{E}=(1 / D)\left[\frac{\sqrt{ } \pi}{12}(k \delta)^{2} I_{1}-(k \delta / 3) I_{2}+\frac{\sqrt{ } \pi}{8} I_{3}\right],
$$

or

$$
\alpha^{E}(\hbar \omega)=\left(\alpha_{0}^{E} / D\right)\left[\frac{\sqrt{ } \pi}{12}(k \delta)^{2} I_{1}-(k \delta / 3) I_{2}+\frac{\sqrt{ } \pi}{8} I_{3}\right]
$$

where

and

$$
\begin{aligned}
& D=\left[\frac{\sqrt{ } \pi}{6}(k \delta)^{2}-4 k \delta / 3+2 \sqrt{ } \pi\right] \\
& I_{1}=\int_{y_{\mathrm{s}}}^{y_{\mathrm{m}}}\left(I / I_{0}\right)\left(y_{\mathrm{s}} / y\right)^{1 / 2}(\mathrm{~d} y / y), \\
& I_{2}=\int_{y_{\mathrm{s}}}^{y_{\mathrm{m}}}\left(I / I_{0}\right)\left(y_{\mathrm{s}} / y\right)^{1 / 2} \ln \left(y / y_{\mathrm{s}}\right)(\mathrm{d} y / y), \\
& I_{3}=\int_{y_{\mathrm{s}}}^{y_{\mathrm{m}}}\left(I / I_{0}\right)\left(y_{\mathrm{s}} / y\right)^{1 / 2}\left\{\ln \left(y / y_{\mathrm{s}}\right)\right\}^{2}(\mathrm{~d} y / y), \\
& y_{\mathrm{s}}=\gamma^{1 / 3} F_{\mathrm{s}}^{-2 / 3}\left(E_{\mathrm{g}}-\hbar \omega\right), \\
& y_{\mathrm{m}}=\gamma^{1 / 3} k^{-2 / 3}\left(E_{\mathrm{g}}-\hbar \omega\right)^{1 / 3},
\end{aligned}
$$

$$
I=I_{0} \text { at } h \omega=E_{\mathrm{g}} \text {. }
$$

\subsection{Mechanical stress}

Thin films are generally characterized by the presence of a large number of defects and the existence of high stress. The polycrystalline films are described by the 


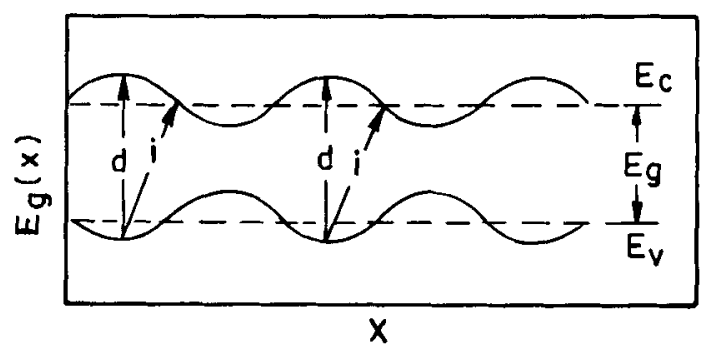

Figure 2. Qualitative picture of deformed valence and conduction band edges of polycrystalline semiconductors.

presence of alternate grains (crystallites) and grain-boundary (amorphous) regions. This would amount to the presence of large stresses in the grain-boundary regions (Szczyrbowski 1979; Klyava 1985; Gavrilenko 1987). Thus, besides the grain-boundary electric field effect, the mechanical stress due to lattice dilation at the grain-boundary regions which may include permanent lattice disorder (in the grain and grainboundary regions) and thermal lattice disorder also influence the electronic structure and hence the below-band-edge optical absorption processes as a whole.

The influence of mechanical stress in the grain boundary region of polycrystalline films has been associated with electrostatic fluctuations of the band edge (figure 2) by several authors (Szczyrbowski 1979; Klyava 1985; Gavrilenko 1987). The basic idea of the above model is the existence of a finite probability of transitions between states lying at different sites in the polycrystalline sample. This will control the specific shape of the absorption tail in the below-band-edge absorption region. Besides these indirect transitions, allowed direct transitions may also occur simultaneously in these polycrystalline films. But the latter will predominate only in the photon energy region $\hbar \omega>\bar{E}_{\mathrm{g}} . \bar{E}_{\mathrm{g}}$ is the average value of the fluctuating $E_{\mathrm{g}}$ in the grain and grain-boundary regions. So we will not consider the contribution from the latter on the tail of the optical absorption.

In order to calculate the absorption due to mechanical stress, we consider first the variation of $E_{\mathrm{g}}$ in various sites of the specimen by the following distribution function (Szczyrbowski 1979, 1981):

$$
D\left(E_{\mathrm{g}}\right) \sim \exp \left[\frac{-\left(E_{\mathrm{g}}-\bar{E}_{\mathrm{g}}\right)^{2}}{2 \sigma^{2}}\right],
$$

where $\sigma^{2}=E_{\mathrm{g}}^{2} \bar{\Delta}^{2}, \bar{\Delta}^{2} \sim a / 4 L_{\mathrm{c}}, L_{\mathrm{c}}$ being the characteristic length over which $E_{\mathrm{g}}$ is assumed to be constant and $a$ the average lattice constant. For polycrystalline material, the characteristic length is set equal to the Debye screening length $\left(\lambda_{d}\right)$ in this case. Physically, the parameter $\bar{\Delta}$ is a relative local and thermal fluctuating dilation constant and depends on the grain size and shape of the polycrystalline film and its lattice temperature.

Now, the general form of the absorption coefficient $\alpha^{M}(\hbar \omega)$ due to mechanical stress may be expressed as

$$
\alpha^{M}(\hbar \omega) \sim \int_{\hbar \omega}^{\alpha} P(E) D\left(E_{\mathrm{g}}\right) \mathrm{d} E_{\mathrm{g}},
$$


where $D\left(E_{\mathrm{g}}\right) \mathrm{d} E_{\mathrm{g}}$ is proportional to the number of microcrystallites per unit volume and $P(E)$ is the probability of indirect transitions within the region of the sample in which the energy gaps are between $E_{\mathrm{g}}$ and $E_{\mathrm{g}}+\mathrm{d} E_{\mathrm{g}}$.

Assuming the form of $P(E)$ as given by Szczyrbowski $(1979,1981)$ :

$$
P(E)=P_{0} \exp \left[\left(\hbar \omega-E_{\mathrm{g}}\right) / E^{\prime}\right],
$$

where $E^{\prime}=3 \sqrt{ }\left[\left(\hbar^{2} k^{2} \sigma^{2}\right) / 6 m_{\mathrm{e}}\right]$ and substituting (9) in (10), the final form of $\alpha^{M}(\hbar \omega)$ after simplification becomes

$$
\alpha^{M}(\hbar \omega)=\alpha_{0}^{M} \exp \left[\left(\hbar \omega-E_{0}\right) / E^{\prime}\right] \Phi(\hbar \omega),
$$

where $\alpha_{0}^{M}$ is the value of $\alpha^{M}(\hbar \omega)$ at $E_{\mathrm{g}}, E_{0}=E_{\mathrm{g}}-\sigma^{2} / 2 E^{\prime}$, and $\Phi(\hbar \omega)$ is a slowly varying function written as

$$
\Phi(\hbar \omega)=E_{\mathfrak{g}}^{-1} \int_{h \omega}^{x} \exp \left[-\left(\sigma^{2} / 2 E^{\prime 2}\right)\right] \mathrm{d} E_{\mathfrak{g}} .
$$

In the photon energy region $\hbar \omega \leqslant E_{\mathrm{g}}$ which is of special interest to us here, the above function $\Phi(h \omega)$ is almost constant and its explicit form may be given by

with

$$
\Phi(\hbar \omega)=\left(\frac{\hbar^{2} k^{2}}{6 m_{\mathrm{e}}}\right) E_{\mathrm{g}}^{-1} \exp \left[-\left(\bar{\Delta}^{2}\right)^{1 / 3} / 2\right] \int_{x_{\min }}^{x_{\max }} \exp \left(-x^{2 / 3}\right) \mathrm{d} x,
$$

$$
x_{\min }=6 m_{\mathrm{e}} \hbar \omega / \hbar^{2} k^{2} \text { and } x_{\max }=6 m_{\mathrm{e}} E_{\mathrm{g}} / \hbar^{2} k^{2} .
$$

In a real situation, the inclusion of finite grain size distribution as given by (6) will modify the expression of $\alpha^{M}(\hbar \omega)$, i.e. (12), as

$$
\alpha^{M}(\hbar \omega)=\alpha_{0}^{M} \exp \left[\left(\hbar \omega-E_{\mathrm{g}}\right) / C_{0}\right] \theta(R) \Phi(R, h \omega),
$$

where $\theta(R) \sim \int_{R_{\min }}^{R_{\max }} \exp \left(-X_{0}\right) R^{2} \mathrm{~d} R$, with $\left.C_{0}=\left[\left(\hbar^{2} k^{2} E_{\mathrm{g}}^{2}\right) / 48 m_{\mathrm{e}}\right)\right]^{1 / 3}, \Phi(R, \hbar \omega) \sim$ $\Phi(\hbar \omega)$, and $X_{0}=(R / \delta)^{2}-(R / a)^{1 / 3}-\frac{E_{\mathrm{g}}^{2}}{16 C_{0}\left(E_{\mathrm{g}}-\hbar \omega\right)}(a / R)^{1 / 3}$. The inclusion of the grain distribution has a negligible influence on $\alpha^{M}(h \omega)$.

By using (8) and (15), one can get the total below-sub-band-edge absorption $\alpha=\left(\alpha^{E}+\alpha^{M}\right)$ and hence $\left(\alpha / \alpha_{0}\right)$ due to grain-boundary effects. The best fit of this simple theoretical model to the experimental plots will provide meaningful information on the grain-boundary potentials, density of trap states, carrier concentration, etc of polycrystalline semiconductor films.

The above exercise becomes meaningful when one can obtain the absorption data quite accurately. The films being polycrystalline, surface roughness will play a dominant role in determining transmittance and reflectance in the films. Scattered transmittance $\left(T_{\text {scatter }}\right)$ and diffuse reflectance $\left(R_{\text {diff }}\right)$ are sure to make a significant contribution (Bennett and Bennett 1967; Tuttle et al 1989; Bhattacharyya et al $1992 \mathrm{~b})$ in the evaluation of the absorption coefficient $(\alpha)$. In recent communications (Bhattacharyya et al 1992a,b), we could show that, by measuring reflectances of a film from the film side $(R)$ and the substrate side $\left(R_{1}\right)$, one can calculate the absorption 
data with sufficient accuracy so as to incorporate (Bhattacharyya et al 1992a) the behaviour of the absorption versus wavelength as demanded by the theory. We could also show (Bhattacharyya et al 1992b) that by measuring $R$ and $R_{\text {diff }}$, one can obtain the grain size and grain distribution quite accurately. This will mean that just by measuring the reflectances $R, R_{1}$ and $R_{\text {diff }}$ as depicted in the previous communications (Bhattacharyya et al 1992a,b) one can measure the absorption coefficient and the grain size quite accurately to use the expressions (8) and (15) for deriving meaningful information on the grain-boundary potential, density of trap states and carrier concentration of a high-resistivity semiconductor film.

\section{Experimental observations}

Figure 3 shows the variation of $\alpha / \alpha_{0}$ versus $\left(E_{\mathrm{g}}-\hbar \omega\right)$ for $\mathrm{ZnTe}$ film deposited onto glass substrate at a temperature of $553 \mathrm{~K}$ by the hot wall evaporation technique (Maiti et al 1994). The individual contributions from the electric field effects (8) and the mechanical effects (15) are shown in figure 3 along with the total theoretical $\alpha / \alpha_{0}$ values. For theoretical simulation of the below-band-edge absorption curve, we used the values of different parameters as follows:

$m_{\mathrm{e}}=0.10 m_{0} ; m_{\mathrm{h}}=0.6 m_{0} ; \Delta_{0}=0.91 \mathrm{eV} ; a=6.085 \AA$ (obtained from XRD traces) and $\bar{R}=0.138 \mathrm{~nm}$ (derived from reflectance measurements, Bhattacharyya et al 1992b). The values of optical constants, $E_{\mathrm{g}}=2.275 \mathrm{eV}, n=2.7$ and $\alpha=9512 \mathrm{~cm}^{-1}$ at photon energy $h v=E_{\mathrm{g}}$, used here were reported in Maiti et al (1994).

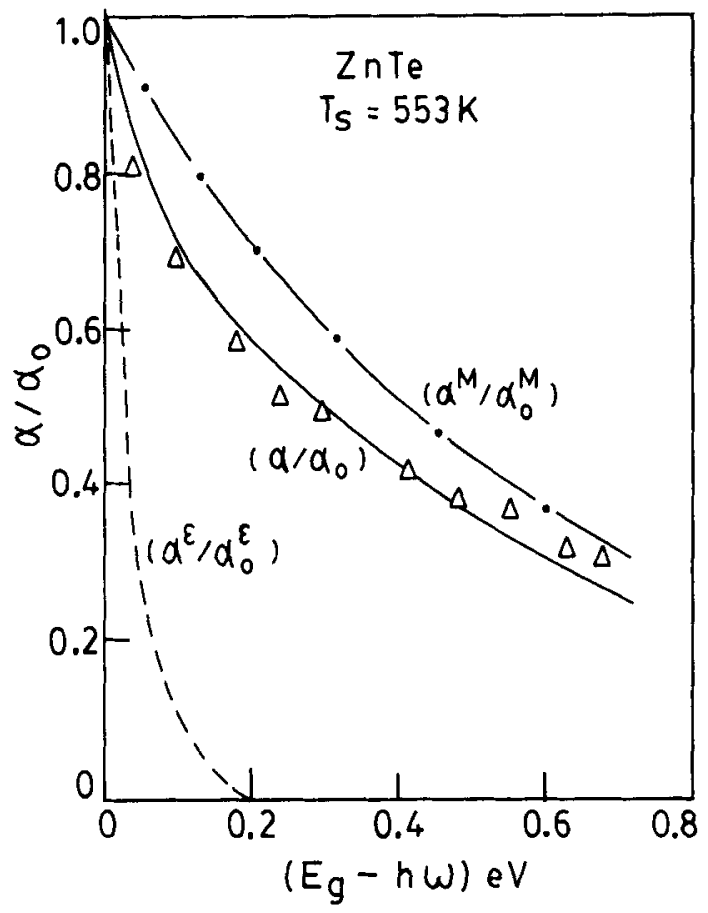

Figure 3. $\alpha / \alpha_{0}$ vs $\left(E_{\mathrm{B}}-\hbar \omega\right)$ plots for ZnTe films: $\Delta$, experimental points;,$--- \alpha^{E} / \alpha_{0}^{E}$; $-\cdot \cdot-, \alpha^{M} / \alpha_{0}^{M} ; \ldots$, total $\alpha / \alpha_{0}\left(\alpha=\alpha^{E}+\alpha^{M}\right)$. 
In the course of the best theoretical fitting procedure, we first chose some guess values of $F_{\mathrm{s}}$ and $k$ for generating the contribution from electric field while a guess value for $\lambda_{\mathrm{d}}$ was used for evaluating the contribution of the effect due to mechanical stress. Then by using (8) and (15) and by adopting an iterative technique, we could easily obtain the best simulated value for the fit of the experimental data.

It can be observed that the contributions arising from the existence of mechanical stresses in polycrystalline semiconductor films are quite significant in describing the shape of the absorption curve beyond the absorption edge. The fit of the experimental data $\left(\alpha / \alpha_{0}\right)$ with the theoretical values of $\left(\alpha / \alpha_{0}\right)$ evaluated by considering the contributions from both the grain-boundary effect and mechanical stress is remarkable. The density of trap states $\left(Q_{1}\right)$ and the barrier height $\left(E_{\mathrm{b}}\right)$ computed from the above fit for $\mathrm{ZnTe}$ films were found to be $5.013 \times 10^{12} \mathrm{~cm}^{-2}$ and $0.205 \mathrm{eV}$ respectively, which compare well with those obtained earlier (Maiti et al 1994).

\section{Conclusions}

The effect of grain boundaries on the optical absorption below the band edge in polycrystalline semiconductor films has been considered. It was observed that the band tailing could be effectively explained by considering the effects of inherent electric field and mechanical stress simultaneously present at the grain boundaries. A realistic approach to the above problem was conceived by incorporating the actual surface roughness and grain distribution present in the films. The above theory was successfully applied to generate information on the barrier height and density of trap states present at the grain boundaries of $\mathrm{ZnTe}$ films.

\section{References}

Baccarani G, Ricco B and Spadini G 1978 J. Appl. Phys. 495565

Bennett H E and Bennett J M 1967 in Physics of thin films (eds) G Hass and R E Thun (New York: Academic Press) vol. 4, p. 1

Bhattacharyya D, Chaudhuri S and Pal A K 1992a Vacuum 43313

Bhattacharyya D, Bhattacharyya S K, Chaudhuri S and Pal A K 1992b Vacuum 431201

Bhattacharyya D, Chaudhuri S and Pal A K 1993 Vacuum 44797

Bugnet P 1974 Rev. Phys. Appl. 9447

Bujatti M and Marcelja F 1972 Thin Solid Films 11249

Dow J P and Redfield D 1970 Phys. Rev. B1 3358

Dow J P and Redfield D 1972 Phys. Rev. B5 594

Gavrilenko V I 1987 Phys. Status Solidi (b) 139457

Kamins T I 1971 J. Appl. Phys. 424357

Klyava Y G 1985 Sov. Phys. Solid State 27816

Maiti B, Gupta P, Chaudhuri S and Pal A K 1994 Thin Solid Films 239104

Redfield D 1963 Phys. Rev. 130916

Redfield D 1965 Phys. Rev. A140 2056

Seto J Y W 1975 J. Electrochem. Soc. 122701

Seto J Y W 1976 J. Appl. Phys. 465247

Szczyrbowsky J and Czapla A 1977 J. Appl. Phys. 46127

Szczyrbowski J J 1979 Phys. Status Solidi (b) 10596

Szczyrbowski J J 1981 Phys. Status Solidi (b) 105515

Tuttie J R, Albin D, Matson R J and Noufi R 1989 J. Appl. Phys. 664408

Volger J 1950 Phys. Rev. 91023 